IITAP-99-013

hep-ph/9911228

\title{
The Next-to-Leading BFKL Pomeron with Optimal Renormalization ${ }^{a}$
}

\author{
Victor T. Kim ${ }^{\ddagger \&}$, Lev N. Lipatov \\ and \\ Grigorii B. Pivovarov ${ }^{\S \&}$ \\ ¥ : St.Petersburg Nuclear Physics Institute, 188350 Gatchina, Russia \\ $\S$ : Institute for Nuclear Research, 117312 Moscow, Russia \\ \& : International Institute of Theoretical and Applied Physics, \\ Iowa State University, Ames, IA 50011, USA
}

\begin{abstract}
The next-to-leading order (NLO) corrections to the BFKL equation in the BLM optimal scale setting are briefly discussed. A striking feature of the BLM approach is rather weak $Q^{2}$-dependence of the Pomeron intercept, which might indicate an approximate conformal symmetry of the equation. An application of the NLO BFKL resummation for the virtual gamma-gamma total cross section shows a good agreement with recent L3 data at the CERN LEP2.
\end{abstract}

The Balitsky-Fadin-Kuraev-Lipatov (BFKL) 1 . 1 resummation of energy logarithms is anticipated to be an important tool for exploring the high-energy limit of QCD. Namely, the highest eigenvalue, $\omega^{\max }$, of the BFKL equation 1 is related to the intercept of the Pomeron which in turn governs the highenergy asymptotics of the cross sections: $\sigma \sim s^{\alpha_{I P}-1}=s^{\omega^{\max }}$. The BFKL Pomeron intercept in the leading order (LO) turns out to be rather large: $\alpha_{I P}-1=\omega_{L O}^{\max }=12 \ln 2\left(\alpha_{S} / \pi\right) \simeq 0.55$ for $\alpha_{S}=0.2$; hence, it is very important to know the next-to-leading order (NLO) corrections. In addition, the LO BFKL calculations have restricted phenomenological applications because, e.g., the running of the QCD coupling constant $\alpha_{S}$ is not included, and the kinematic range of validity of LO BFKL is not known.

Recently the NLO corrections the BFKL resummation of energy logarithms were calculated; see Refs. 뇓 and references therein. The NLO corrections 4 to the highest eigenvalue of the BFKL equation turn out to be negative and even larger than the LO contribution for $\alpha_{S}>0.16$.

It should be stressed that the NLO calculations, as any finite-order perturbative results, contain both renormalization scheme and renormalization

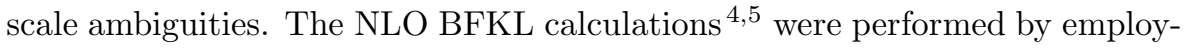

${ }^{a}$ presented by V.T. K. at the VIIIth International Blois Workshop, June 28 - July 2, 1999, Institute for High Energy Physics, Protvino, Russia, to appear in the Proceedings 
ing the modified minimal subtraction scheme $(\overline{\mathrm{MS}})$ to regulate the ultraviolet divergences with arbitrary scale setting.

In the recent work 6 it was found that the renormalization scheme dependence of the NLO BFKL resummation of energy logarithms 4 is not strong, i.e., value of the NLO BFKL term is practically the same in the known renormalization schemes. To resolve the renormalization scale ambiguity due to the large NLO BFKL term 1 setting $\mathrm{U}$ has been utilized. The BLM optimal scale setting effectively resums the conformal-violating $\beta_{0}$-terms into the running coupling in all orders of the perturbation theory.

It was shown 6 that the reliability of QCD predictions for the effective intercept of the BFKL Pomeron at NLO evaluated at the BLM scale setting within the non-Abelian physical schemes, such as the momentum space subtraction

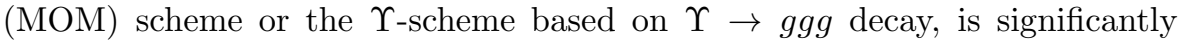
improved compared to the $\overline{\mathrm{MS}}$-scheme result $9 \mathrm{10}$.

One of the striking features of the analysis 6 is that the NLO value for the intercept of the BFKL Pomeron, improved by the BLM procedure, has a very weak dependence on the gluon virtuality $Q^{2}: \alpha_{I P}-1=\omega_{N L O}^{\max }=\simeq 0.13-0.18$ at $Q^{2}=1-100 \mathrm{GeV}^{2}$. It arises as a result of fine-tuned compensation between the LO and NLO contributions. The minor $Q^{2}$-dependence obtained leads to approximate conformal invariance.

As a phenomenological application of the NLO BFKL improved by BLM procedure one can consider the gamma-gamma scattering 11. This process is attractive because it is theoretically more under control than the hadronhadron and lepton-hadron collisions, where non-perturbative hadronic structure functions are involved. In addition, in the gamma-gamma scattering the unitarization (screening) corrections due to multiple Pomeron exchange would be less important than in hadron collisions.

The gamma-gammacross sections with the BFKL resummation in the LO was considered in 2, 22, 13. In the NLO BFKL case one should obtain a formula analogous to LO BFKL 11 . While exact NLO impact factor of gamma is not known yet, one can use the LO impact factor of 12 assuming that the main energy-dependent NLO corrections come from the NLO BFKL subprocess rather than from the impact factors 11 .

In Fig. 1 the comparison of BFKL predictions for LO and NLO BFKL improved by the BLM procedure with L3 data 44 from CERN LEP2 is shown. The different curves reflect uncertainty with the choice of the Regge scale parameter which defines the beginning of the asymptotic regime. For present calculations two variants have been choosen $s_{0}=Q^{2} / 2$ and $s_{0}=2 Q^{2}$, where $Q^{2}$ is virtuality of photons. One can see from Fig. 1 that the agreement of 


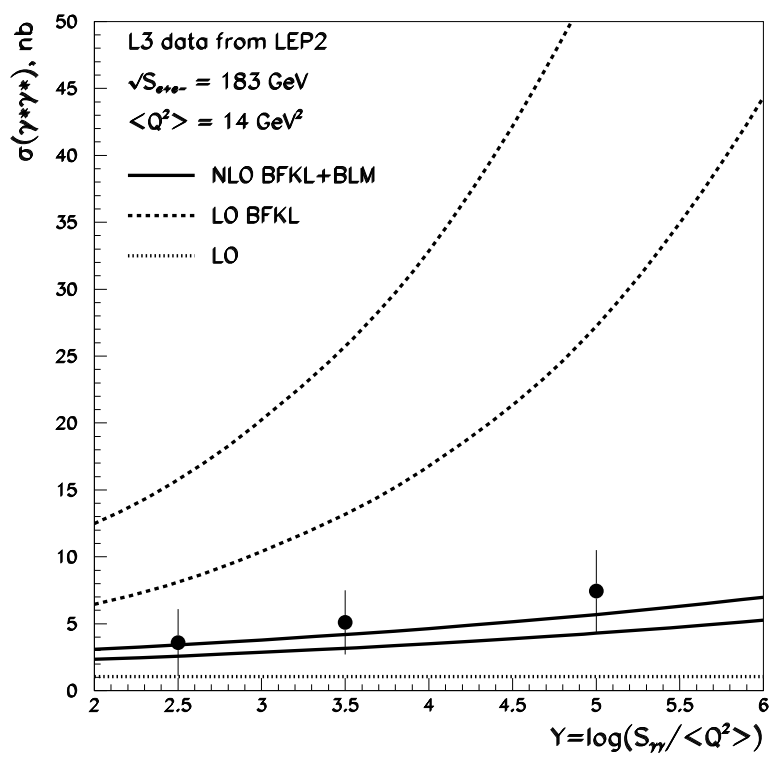

Figure 1: Virtual gamma-gamma total cross section by the NLO BFKL Pomeron within BLM approach vs L3 Collaboration data at energy $183 \mathrm{GeV}$ of $e^{+} e^{-}$collisions. Solid curves correspond to NLO BFKL in BLM; dashed: LO BFKL and dotted: LO contribution. Two different curves are for two different choices of the Regge scale: $s_{0}=Q^{2} / 2, s_{0}=2 Q^{2}$

the NLO BFKL improved by the BLM procedure is reasonably good at LEP2 energy $\sqrt{s_{e^{+} e^{-}}}=183 \mathrm{GeV}$. One can notice also that sensitivity of the NLO BFKL results to the Regge parameter $s_{0}$ is much smaller than in the case of the LO BFKL.

It was shown in Refs. 15,16 that the unitarization corrections in hadron collisions can lead to higher value of the (bare) Pomeron intercept than the effective intercept value, Since the hadronic data fit yields about 1.1 for the effective intercept value 16, then the bare Pomeron intercept value should be above this value. Therefore, assuming small unitarization corrections in the gamma-gamma scattering at large $Q^{2}$ one can accomodate the NLO BFKL Pomeron intercept value 1.13 - 1.18 in the BLM optimal scale setting along with larger unitarization corrections in hadronic scattering 15 , where they can lead to a smaller effective Pomeron intercept value about 1.1 for hadronic collisions. 
Another possible application of the BFKL approach can be the collision energy dependence of the inclusive single jet production 17 .

There have been a number of recent papers which analyze the NLO BFKL predictions in terms of rapiditycorrelations 18 angle-ordering 19 , double transverse momentum logarithms 2021.22, an additional $\log (1 / x)$ enhancement 23 and BLM scale setting for deep inelastic structure functions 24 . Obviously, a lot of work should be done to clarify the proper expansion parameter for BFKL regime and, also the relation between those papers and the result of the present BLM approach. To confirm the result of ${ }^{-}$the independent NLO calculations (see 25 and references therein) for BFKL resummation are desirable.

To conclude, we have shown that the NLO corrections to the BFKL equation for the QCD Pomeron become controllable and meaningful provided one uses physical renormalization scales and schemes relevant to non-Abelian gauge theory. BLM optimal scale setting automatically sets the appropriate physical renormalization scale by absorbing the non-conformal $\beta$-dependent coefficients. The strong renormalization scheme and scale dependence of the NLO corrections to BFKL resummation then largely disappears. A striking feature of the NLO BFKL Pomeron intercept in the BLM approach is its very weak $Q^{2}$-dependence, which provides approximate conformal invariance. The NLO BFKL application to the total gamma-gamma cross section shows a good agreement with the L3 Collaboration data at CERN LEP2 energies.

VTK thanks the Organizing Committee of the VIIIth Blois Workshop at Institute for High Energy Physics, Protvino for their warm hospitality. This work was supported in part by INTAS, Grant No. INTAS-97-31696.

1. E. A. Kuraev, L. N. Lipatov and V. S. Fadin, ZhETF 71, 840 (1976) [Sov. JETP 44, 443 (1976)]; ibid. 72, 377 (1977) [45, 199 (1977)]

2. I. I. Balitsky and L. N. Lipatov, Yad. Fiz. 28, 1597 (1978) [Sov. J. Nucl. Phys. 28, 822 (1978)]

3. L. N. Lipatov, Phys. Rept. C286, 131 (1997)

4. V. S. Fadin and L. N. Lipatov, Phys. Lett. 429B, 127 (1998)

5. G. Camici and M. Ciafaloni, Phys. Lett. 430B, 349 (1998)

6. S. J. Brodsky, V. S. Fadin, V. T. Kim, L. N. Lipatov and G. B. Pivovarov, Pisma ZhETF 70,161 (1999) [JETP Lett.70,155(1999)], hep-ph/9901229

7. S. J. Brodsky, G. P. Lepage and P. B. Mackenzie, Phys. Rev. D28, 228 (1983)

8. L. N. Lipatov, ZhETF 90, 1536 (1986) [Sov. JETP 63, 904 (1986)]; in Perturbative QCD, ed. A.H. Mueller (World Scientific, Singapore, 1989) p. 411; R. Kirschner and L. Lipatov, Zeit. Phys. C45, 477 (1990)

9. D. A. Ross, Phys. Lett. 431B, 161 (1998); Yu. V. Kovchegov and A. H. Mueller, ibid. 439B, 428 (1998); N. Armesto, J. Bartels and 
M. A. Braun, ibid. 442B, 459 (1998); E. M. Levin, Nucl. Phys. B545, 481 (1999)

10. J. Blümlein, V. Ravindran, W. L. van Neerven and A. Vogt, Proc. DIS and QCD (DIS98), Brussels, Belgium, April 4-8, 1998, eds. Gh. Coremans and R. Roosen (World Scientific, Singapore, 1998) p. 211, hepph/9806368; R. D. Ball and S. Forte, ibid., p. 770, hep-ph/9805315

11. S. J. Brodsky, V. S. Fadin, V. T. Kim, L. N. Lipatov and G. B. Pivovarov, in preparation

12. S. J. Brodsky, F. Hautmann, D. E. Soper, Phys. Rev. D56, 6957 (1997); Phys. Rev. Lett. 78, 803 (1997), (E) 79, 3544 (1997)

13. J. Bartels, A. De Roeck and H. Lotter, Phys. Lett. 389B, 742 (1996); A. Białas, W. Czyż and W. Florkowski, Eur. Phys. J. C2, 683 (1998); M. Boonekamp, A. De Roeck, C. Royon and S. Wallon, Nucl. Phys. B555, 540 (1999); J. Kwieciński and L. Motyka, Acta Phys. Pol. B30, $1817(1999)$

14. L3 Coll., M. Acciari et al., Phys. Lett. 453B, 333 (1999)

15. A. B. Kaidalov, L. A. Ponomarev and K. A. Ter-Martirosyan, Yad. Fiz. 44, 722 (1986) [Sov. J. Nucl. Phys. 44, 468 (1986)]

16. J. R. Cudell, V. Ezhela, K. Kang, S. Lugovsky and N. Tkachenko, BROWN-HET-1184, 1999, hep-ph/9908218; J. R. Cudell, K. Kang and S. K. Kim, Phys. Lett. 395B, 311 (1997); J. R. Cudell, A. Donnachie and P. V. Landshoff, Phys. Lett. 448B, 281 (1999)

17. V. T. Kim and G. B. Pivovarov, Phys. Rev. D57, 1341 (1998)

18. V. S. Fadin and L. N. Lipatov, Proc. Theory Institute on Deep Inelastic Diffraction, ANL, Argonne, September 14-16, 1998; C. R. Schmidt, Phys. Rev. D60, 074003 (1999); J. R. Forshaw, D. A. Ross and A. Sabio Vera, Phys. Lett. 455B, 273 (1999)

19. S. Catani, F. Fiorani and G. Marchesini, Nucl. Phys. B336, 18 (1990); M. Ciafaloni, Nucl. Phys. B296, 49 (1988)

20. B. Andersson, G. Gustafson and J. Samuelsson, Nucl. Phys. B467, 443 (1996); B. Andersson, G. Gustafson and H. Kharraziha, Phys. Rev. D57, 5543 (1998)

21. G. Salam, JHEP 9807, 019 (1998)

22. M. Ciafaloni, D. Colferai and G. P. Salam, DFF-338-5-99, 1999, hepph/9905566; M. Ciafaloni and D. Colferai, Phys. Lett. 452B, 372 (1999)

23. R. D. Ball and S. Forte, Edinburgh-99/5, 1999, hep-ph/9906222

24. R. S. Thorne, Phys. Rev. D60, 054031 (1999)

25. Z. Bern, V. Del Duca, W. B. Kilgore and C. R. Schmidt, BNL-HET-99-6, 1999, hep-ph/9903516 